\title{
Acute ischemic stroke as a complication of Stanford type A acute aortic dissection: a review and proposed clinical recommendations for urgent diagnosis
}

\author{
Masatoshi Koga ${ }^{1} \cdot$ Yasuyuki Iguchi $^{2} \cdot$ Tomoyuki Ohara $^{3} \cdot$ Yoshio Tahara $^{4} \cdot$ Tetsuya Fukuda $^{5} \cdot$ Teruo Noguchi $^{4}$. \\ Hitoshi Matsuda $^{6} \cdot$ Kenji Minatoya $^{6,7} \cdot$ Kazuyuki Nagatsuka $^{8} \cdot$ Kazunori Toyoda $^{1}$
}

Received: 17 April 2018 / Accepted: 8 June 2018 / Published online: 13 June 2018

(c) The Author(s) 2018

\begin{abstract}
Background Stanford type A acute aortic dissection requires emergency surgery. Because patients with ischemic stroke as a complication of Stanford type A acute aortic dissection do not often complain of chest or back pain, probably due to consciousness disturbance, amnesia, or aphasia, a fatal course following inappropriate intravenous rt-PA therapy and delay of appropriate surgical treatment sometimes occur.

Review and proposed recommendations When treating any suspected stroke patients, emergency services and initial urgent care doctors should always suspect aortic dissection. Even in the absence of chest or back pain, the initial urgent care doctor needs to immediately perform chest contrast $\mathrm{CT}$ if suspecting aortic dissection from blood pressure laterality or upper mediastinal widening on chest X-ray. Whenever aortic dissection cannot be ruled out from initial clinical information, the initial urgent care doctor should evaluate the common carotid artery (CCA). Dissection extension to the CCA or flow abnormality of the CCA is often detected if aortic dissection is a cause of ischemic stroke or transient ischemic attack. Head $\mathrm{CT}$ or MRI including vascular imaging is preferable. D-dimer should be measured in hospitals where available. As soon as aortic dissection is identified, the initial urgent care doctor needs to consult with cardiovascular surgeons or cardiologists for appropriate treatment.
\end{abstract}

Keywords Intravenous rt-PA therapy $\cdot$ Chest or back pain $\cdot$ Blood pressure laterality $\cdot$ Carotid ultrasonography $\cdot$ D-dimer

Masatoshi Koga

koga@ncvc.go.jp

1 Department of Cerebrovascular Medicine, National Cerebral and Cardiovascular Center, 5-7-1 Fujishirodai, Suita, Osaka 565-8565, Japan

2 Department of Neurology, The Jikei University School of Medicine, Tokyo, Japan

3 Department of Neurology, Kyoto Prefectural University of Medicine, Kyoto, Japan

4 Department of Cardiovascular Medicine, National Cerebral and Cardiovascular Center, Suita, Japan

5 Department of Radiology, National Cerebral and Cardiovascular Center, Suita, Japan

6 Department of Cardiovascular Surgery, National Cerebral and Cardiovascular Center, Suita, Japan

7 Department of Cardiovascular Surgery, Kyoto University, Kyoto, Japan

8 Department of Neurology, National Cerebral and Cardiovascular Center, Suita, Japan

\section{Introduction}

By about 2 years after the approval of thrombolytic therapy with alteplase (recombinant tissue-type plasminogen activator: rt-PA) for acute ischemic stroke, 10 acute ischemic stroke patients as a complication of acute aortic dissection had died after rt-PA use, prompting the Japan Stroke Society to issue a warning "to evaluate the indication carefully in patients who may have concomitant thoracic aortic dissection or aneurysm" [1]. In 2008, the Japanese Journal of Stroke published a report regarding cautions of aortic dissection in intravenous thrombolytic therapy with alteplase which proposed "to keep in mind the potential presence of acute aortic dissection as a cause of ischemic stroke", the "importance of basic evaluations and observations", "cooperation with paramedics", "laterality of limb pulse palpation", and the "usefulness of carotid ultrasonography" $[1,2]$. Given the present state after more than 10 years since the approval of rt-PA, we reviewed related articles and researches on acute ischemic stroke as a complication 
of Stanford type A acute aortic dissection (acute type A aortic dissection: ATAAD) and proposed recommendations for urgent diagnosis in a review article in the Japanese Journal of Stroke (Japanese) [3] and updated in this review article.

\section{Ischemic stroke as a complication of Stanford type A acute aortic dissection}

The annual incidence of acute aortic dissection in Japan is approximately 3 per 100,000 population [4]. About 5000 aortic operations for ATAAD are performed nationwide in Japan in 2014 [5]. ATAAD involves dissection of the ascending aorta and requires emergency surgery [6-16]. Without surgical treatment, the outcome is often fatal, with the mortality rate increasing by $1-2 \%$ every hour in the early stage of onset and reaching $50 \%$ or greater among admitted patients. Depending on the extent of aortic dissection extension, it may cause various organ impairments and clinical symptoms; about $6 \%$ of the patients have concomitant ischemic stroke or transient ischemic attack (TIA) due to extension to the cervicocerebral arteries or emboli from the site of dissection [6, 17]. The most classical clinical manifestation of ATAAD is chest or back pain; however, only about half of the patients with ischemic stroke have such a complaint [18-20], probably due to the presence of cortical symptoms such as amnesia or aphasia and/or consciousness disturbance [18]. Issues in treating patients with ischemic stroke as a complication of ATAAD are "fatal course following inappropriate intravenous rt-PA therapy" and "delay of appropriate surgical treatment" due to a delayed diagnosis of aortic dissection [19-22]. The complication of ischemic stroke has been considered a predictor of a poor prognosis $[11,23]$; however, with early diagnosis of ATAAD and appropriate surgical treatment, concomitant neurological symptoms have been reported to be associated with no increase in mortality [18, 24-26]. In ATAAD, temporary occlusion of the aortic arches or cervicocerebral arteries may occur depending on the development or extension of dissection; thus, physicians must note that neurological symptoms may frequently disappear or vary [27]. Moreover, patients may show a combination of various neurological manifestations, such as hemiplegia, consciousness disturbance, syncope, convulsion, amnesia, spinal cord ischemia, and peripheral neuropathy [27].

\section{Clinical characteristics of ischemic stroke as a complication of Stanford type A acute aortic dissection}

Among 226 ATAAD patients admitted to the National Cerebral and Cardiovascular Center (NCVC) between 2007 and 2013, 23 (10\%; female, 58\%; 74 \pm 12 years of age) had ischemic stroke [19]. Their clinical characteristics are shown in Table 1. Stroke physicians provided the initial care to most of the patients (91\%) with ischemic stroke as a complication of ATAAD, with cardiologists providing the care to the rest of the patients $(9 \%)$. Thirteen patients (57\%) were potential candidates for intravenous rt-PA therapy with alteplase in the absence of ATAAD. Diagnosis of ATAAD was delayed for 1 patient, who died after receiving the intravenous rt-PA therapy. Consciousness disturbance was present in 18 patients (78\%), and complaints of chest or back pain were absent in 12 patients (52\%). Common carotid artery occlusion or an intimal flap was detected on carotid ultrasonography in 18 of 20 patients (90\%); high D-dimer level ( $\geq 6.9 \mu \mathrm{g} / \mathrm{ml})$ [28], in 18 of 23 patients (78\%); left hemiplegia, in 17 of 23 patients (74\%); upper limb systolic blood pressure (SBP) laterality ( $\geq 20 \mathrm{mmHg}$ ), in 15 of 21 patients (71\%); and upper mediastinal widening on the chest X-ray, in 10 of 15 patients (67\%).

A study of consecutive 1637 patients who came to NCVC for suspected stroke between January 2012 and January 2013 found a total of 5 patients with ischemic stroke as a complication of ATAAD, accounting for $0.31 \%$ of ambulancetransported stroke-suspected patients and $1.09 \%$ of 457 acute ischemic stroke patients $(1.70 \%$ of those with an onset-todoor time of no more than $4 \mathrm{~h}$ ) [20]. In all patients who had ischemic stroke as a complication of ATAAD, complaints of chest or back pain were absent, and the right upper limb SBP was $\leq 110 \mathrm{mmHg}$. Common carotid artery intimal flap or occlusion occurred in all 4 patients who underwent carotid ultrasonography. Two patients (40\%) died immediately upon arrival at the center, and the other 3 patients (60\%) underwent emergency prosthetic vessel replacement, followed by complete independence in daily living and discharge.

A comparison between 17 consecutive patients admitted to NCVC for ischemic stroke/TIA as a complication of ATAAD who underwent MRI/MRA between January 2009 and December 2015 and 249 consecutive patients admitted to NCVC for ischemic stroke/TIA without ATAAD who underwent MRI/MRA between January 2013 and December 2013, showed that ATAAD patients had infarcts more frequently in the right anterior cerebral artery (ACA) territory (18 vs. $2 \%, p=0.007)$ and the right middle cerebral artery (MCA) territory (71 vs. $29 \%, p<0.001$ ) [29]. On the MRA image, the ATAAD patients more frequently had poor visualization of the right internal carotid artery (ICA) (47 vs. 6\%, $p<0.001)$. After adjustment for sex, age, and confounding factors, right ACA territory infarct (odds ratio (OR) 12.2, 95\% confidence interval (CI) 1.4-119.4), right MCA territory infarct (OR 4.9, 95\% CI 1.0-25.0), and poor visualization of the right ICA (OR 18.1, 95\% CI 4.0-101.9) were more common in AAD patients.

A comparison between 24 consecutive patients admitted to NCVC for ischemic stroke/TIA as a complication of 
Table 1 Characteristics of patients with ischemic stroke as a complication of Stanford type A acute aortic dissection

\begin{tabular}{ll}
\hline & $n=23$ (20 ischemic strokes/3 TIAs) \\
\hline Age (years), mean \pm SD & $74 \pm 12$ \\
Male, $n(\%)$ & $12(52)$ \\
Chest/back pain, $n(\%)$ & $11(48)$ \\
Clinical signs & $18(78)$ \\
Consciousness disturbance, $n(\%)$ & $21(91)$ (right, 4/left, 17) \\
Hemiplegia, $n(\%)$ & $10(43)$ (right, 8/left, 2$)$ \\
Conjugate deviation, $n(\%)$ & \\
Head imaging examination findings & $9(39)$ \\
Right internal carotid region, $n(\%)$ & $1(4)$ \\
Left internal carotid region, $n(\%)$ & 0 \\
Vertebrobasilar arterial region, $n$ & $3(13)$ \\
2 or more vascular regions, $n(\%)$ & \\
Extension of dissection & $23(100)$ \\
Aortic arch, $n(\%)$ & $23(100)$ \\
Brachiocephalic artery, $n(\%)$ & $19(83)$ \\
Right common carotid artery, $n(\%)$ & $12(52)$ \\
Left common carotid artery, $n(\%)$ & $7(23)$ \\
Left subclavian artery, $n(\%)$ & $2(1-3)$ \\
Median onset-to-door time $(q u a r t i l e)(h)$ & $13(57 \%), 1$ (rt-PA therapy) \\
Intravenous rt-PA therapy candidate ${ }^{\mathrm{a}}$ & \\
\hline
\end{tabular}

Modified data from Ref. [19]

${ }^{\text {a }}$ Patients who met the criteria for intravenous rt-PA therapy except for the presence of Stanford type A acute aortic dissection
ATAAD within $4.5 \mathrm{~h}$ of the onset of neurological symptom between January 2007 and December 2014 and 812 consecutive patients admitted to NCVC for ischemic stroke/ TIA without ATAAD within $4.5 \mathrm{~h}$ of onset between January 2012 and December 2014 showed that a high D-dimer level $(\geq 4.1 \mu \mathrm{g} / \mathrm{ml}$, as calculated based on the receiver operating characteristic curve) is highly sensitive (100\%; specificity, 86\%) for detecting ATAAD and useful for screening, and that carotid ultrasonography is highly specific (99\%; sensitivity, 84\%) and can be used to aid a definitive diagnosis (Table 2) [30]. Carotid ultrasonography showed numerous abnormal findings, particularly from the right $(90 \%)$ common carotid artery to the internal carotid artery, including intimal flaps with an unthrombosed false lumen (50\%), intimal flaps with a thrombosed false lumen $(10 \%)$, and various occlusion patterns (30\%). SBP laterality ( $\geq 17 \mathrm{mmHg}$, as calculated based on the receiver operating characteristic curve) and upper mediastinal widening on the chest X-ray were moderately sensitive and specific, while pericardial effusion collection on transthoracic echocardiography showed poor sensitivity (Table 2) [30].

Table 2 Evaluations and tests to detect Stanford type A acute aortic dissection

\begin{tabular}{|c|c|c|c|c|c|}
\hline & Sensitivity (\%) & Specificity (\%) & PPV (\%) & $\operatorname{NPV}(\%)$ & AUC \\
\hline Upper limb SBP laterality $\geq 17 \mathrm{mmHg}$ & 80 & 75 & 9 & 99 & 0.82 \\
\hline D-dimmer $\geq 4.1 \mu \mathrm{g} / \mathrm{ml}$ & 100 & 86 & 18 & 100 & 0.97 \\
\hline Upper mediastinal ratio $\geq 0.32$ on chest $X$-ray & 75 & 76 & 9 & 99 & 0.80 \\
\hline $\begin{array}{l}\text { Common carotid arterial dissection or pattern of blood flow occlu- } \\
\text { sion on carotid ultrasonography }\end{array}$ & 84 & 99 & 80 & 100 & 0.92 \\
\hline Pericardial effusion collection on transthoracic echocardiography & 43 & 100 & 83 & 98 & 0.72 \\
\hline
\end{tabular}

Modified data from Ref. [30]

$A U C$ area under the curve, $N P V$ negative predictive value, $P P V$ positive predictive value 


\section{Nationwide questionnaire survey}

A nationwide web questionnaire survey (sent in October 2015) of 1517 facilities with which a stroke specialist, cardiologist, critical care physician, or cardiovascular surgeon was affiliated was conducted [3]. Responses were received from 527 physicians responsible for treating acute aortic dissection (35\%) and 545 physicians responsible for treating stroke (36\%). Twenty-eight facilities (5.2\%) indicated in their responses that their emergency service activity protocol includes hospital selection with consideration given to the potential presence of ATAAD in suspected stroke patients (e.g., history taking that includes chest or back pain and evaluation of blood pressure laterality). A total of 430 facilities (82\%) treated 1 or more ATAAD patients (median 6, quartile 3-12) in the past year, and the median number of patients with concomitant ischemic stroke was 1 (0-2), indicating that such morbidity was not rare. The most common clinical department that provided the initial care to "ischemic stroke as a complication of ATAAD" (multiple answers allowed) was neurosurgery (511 physicians), followed by emergency (495 physicians), cardiology (373 physicians), neurology (306 physicians), and cardiovascular surgery (154 physicians), with the so-called stroke physicians accounting for about half of the respondents ( $43 \%$ overall). Fourteen patients with "ischemic stroke as a complication of ATAAD" in 13 facilities $(2.4 \%)$ received intravenous rt-PA therapy with alteplase, and 5 (36\%) died during the acute phase. The most common screening test for ATAAD was contrast CT (94\%), followed by chest X-ray (61\%), transthoracic echocardiography (59\%), and D-dimer (47\%). Carotid ultrasonography was seldom used $(8 \%)$. The frequency of examinations for acute management of ischemic stroke patients was $94 \%$ for chest X-ray, $46 \%$ for bilateral upper limb blood pressure measurement, $26 \%$ for carotid ultrasonography, and $24 \%$ for transthoracic echocardiography. Among the respondents, $88.8 \%$ indicated good cooperation between the initial response department and the cardiovascular surgery department, and $11.2 \%$ indicated a limited or poor cooperation with cardiovascular surgery department.

\section{Questionnaire survey of paramedics}

Devising a pre-transport emergency medical care plan may lead to the selection of an appropriate hospital and appropriate post-transport emergency medical care for patients with "ischemic stroke as a complication of ATAAD". To find out what evaluations or actions could be performed when transporting suspected stroke patients, a questionnaire survey of 154 paramedics who participated in a skill-up lecture (September 2016) by NCVC was conducted [3]. The mean length of experience as a paramedic was $8.5 \pm 7.7$ years, $60 \%$ qualified as an emergency lifesaving technician, and $73 \%$ had experience in transporting patients with ATAAD by ambulance. To the question of what evaluations or actions could be performed before transporting patients to a hospital, 83\% (87\%) (percentages of paramedics with more than 1 year of experience are in parentheses) of the respondents selected "to check for the presence/absence of chest or back pain"; $90 \%$ (94\%), "to investigate radial arterial pulse laterality"; 89\% (94\%), "to measure bilateral upper limb blood pressures"; $96 \%$ (99\%), "to investigate presence/absence of consciousness disturbance or the level of consciousness"; $90 \%$ (95\%), "to investigate presence/absence of changes in consciousness disturbance"; and 84\% (88\%), "to transport the patient to a medical facility capable of diagnosis and treatment of both stroke and ATAAD". In particular, nearly $90 \%$ of paramedics with more than 1 year of experience responded that they can perform all of the procedures described above before transporting patients to a hospital.

\section{To urgently diagnose ischemic stroke as a complication of Stanford type $A$ acute aortic dissection}

The nationwide questionnaire survey showed that the incidence of a fatal course following intravenous rt-PA therapy may not have decreased even after more than 10 years since the approval of rt-PA. We propose the "Guidelines for Urgent Diagnosis of Ischemic Stroke as a Complication of ATAAD" shown in Fig. 1 based on available reports, clinical characteristics, and results from the questionnaire survey of facilities across Japan and the questionnaire survey of paramedics.

Step 1 When triaging suspected stroke patients, paramedics should suspect ATAAD and take the following recommended actions: check for the presence/absence of chest or back pain, investigate radial arterial pulse laterality, and investigate the presence/absence of changes in consciousness disturbance. If pulse laterality is present, and the SBP laterality is $\geq 20 \mathrm{mmHg}$ or the right upper limb SBP is $\leq 110 \mathrm{mmHg}$ based on the simultaneous measurement of bilateral blood pressures, ATAAD should be suspected. When the presence of ATAAD is suspected, paramedics should consider transporting the patient to a medical facility able to diagnose and treat both ATAAD and stroke. The involvement by paramedics in nationwide web questionnaire surveys is still rare, and there has been no investigation into whether pre-hospital activities are useful in detecting this morbidity. Nevertheless, the earliest possible diagnosis of 


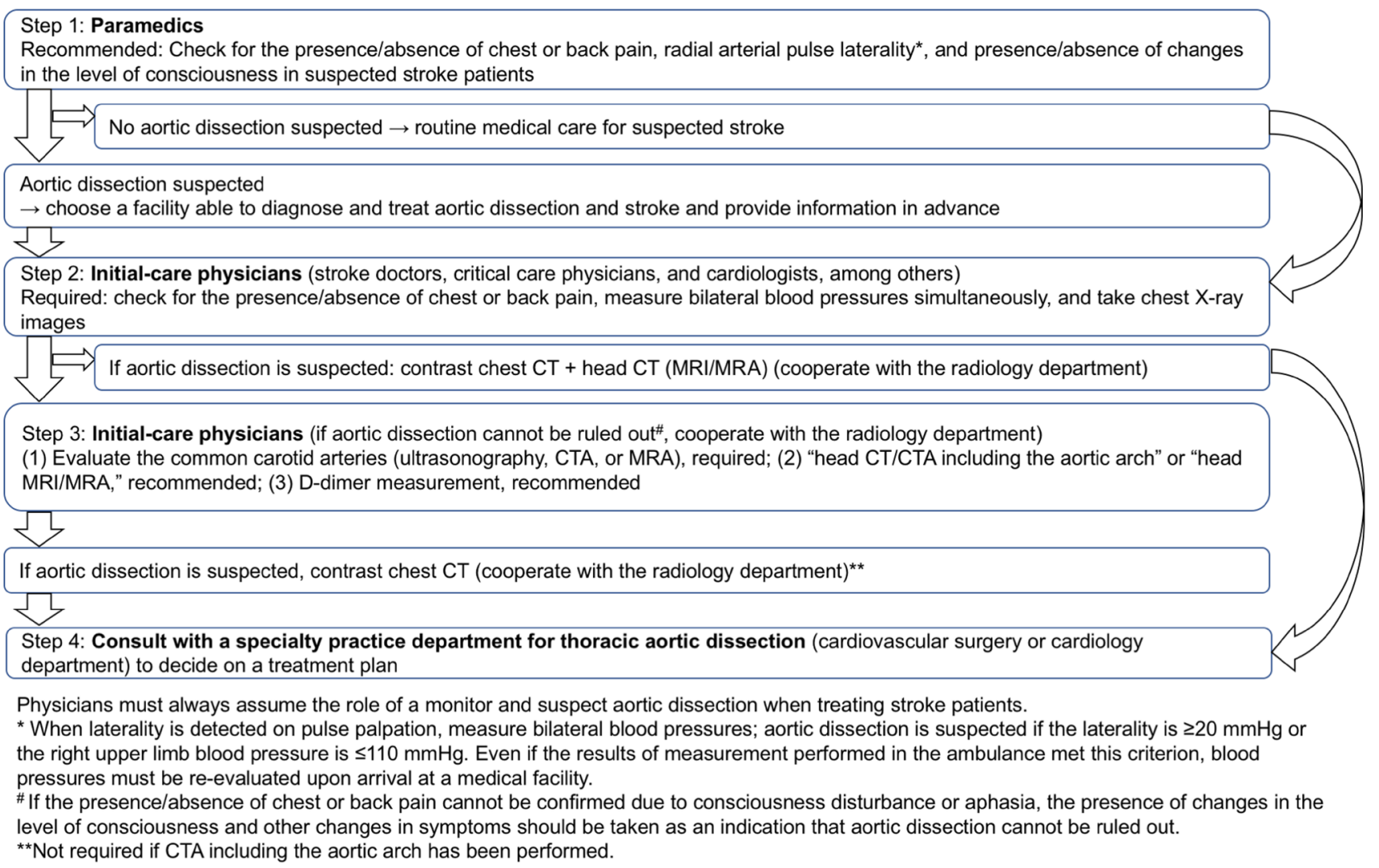

Fig. 1 The four-step guidelines for urgent diagnosis of ischemic stroke caused by Stanford type A acute aortic dissection

this condition greatly impacts the outcome, and the active involvement of paramedics may lead to an appropriate choice of hospital and post-transport critical care.

Step 2 When examining suspected stroke patients, initialcare physicians (approximately half of them are stroke physicians) at a receiving medical facility must keep in mind the potential presence of ATAAD. Confirming the presence/ absence of chest or back pain, simultaneous measurement of bilateral upper limb blood pressures, and chest X-ray are essential to assess whether ATAAD is suspected. When performing essential evaluations and observations, initialcare physicians should keep in mind the presence of such a condition in approximately 1 of 60 ischemic stroke patients $(1.7 \%)$ with an onset-to-door time of no more than $4 \mathrm{~h}$. If ATAAD is suspected based on history taking and examination findings, the patient should promptly undergo a head CT (or MRI/MRA) and a contrast chest CT (in some cases, prioritize contrast chest $\mathrm{CT}$ ) examinations. Stabilizing the general condition should be a priority if the patient is in a state of shock or has an unstable general condition.

Step 3 In cases where ATAAD cannot be ruled out, such as when chest or back pain cannot be confirmed due to consciousness disturbance, amnesia, or aphasia, when changes in the consciousness level or other neurological signs are present during the emergency room examination, or when the patient shows a combination of various neurological manifestations such as hemiplegia, consciousness disturbance, syncope, convulsion, amnesia, spinal cord ischemia, and peripheral neuropathy, initial-care physicians must perform an evaluation of the common carotid arteries. While the choice of imaging study is dependent on the availability at each facility, carotid ultrasonography is the fastest and simplest examination. Other non or minimally invasive tests include MRA and contrast CT. In most patients with ischemic stroke or transient ischemic attack, ultrasonography can detect the direct extension of the aortic dissection to the common carotid artery (commonly, the right side) or blood flow obstruction (commonly, pattern of occluded blood flow) due to obstruction of blood flow to cervicocerebral arteries by the aortic dissection. The accuracy of a diagnosis on carotid ultrasonography performed by an inexperienced technician or physician may not necessarily be high; thus, it is recommended that a technician or physician experienced in evaluating carotid lesions perform the procedure. It is also recommended that head CT or MRI include an evaluation of the intracranial vessels by computed tomography angiography (CTA) or MRA. Contrast CT allows an evaluation of the region from the aortic arch to the neck and the head 
in a single exposure. Evaluate the D-dimer level if the test is available at the facility. In cases where ATAAD is suspected based on history taking, examination findings, results of imaging studies including cervicocerebral arteries, and/ or a D-dimer level $\geq 4.1 \mu \mathrm{g} / \mathrm{ml}$, the patient should undergo contrast chest CT examination promptly.

Step 4 Upon the diagnosis of ATAAD, promptly consult with a specialty practice department, such as the cardiovascular surgery or the cardiology department, to decide on an appropriate treatment plan.

\section{Conclusions}

Acute stroke requires urgent care; it is often a race against time to initiate treatment. As such, initial-care physicians must constantly keep in mind that approximately $1.7 \%$ of suspected stroke patients have ATAAD. To avoid inappropriate intravenous rt-PA therapy and to initiate surgical treatment as soon as possible, perform a contrast chest CT examination immediately when ATAAD is suspected based on the presence of chest or back pain, blood pressure laterality, and/or upper mediastinal widening. If ATAAD cannot be ruled out, perform an imaging study including the common carotid arteries, and measure the D-dimer level; if ATAAD is suspected, perform a contrast CT examination. The proposed guidelines still need to undergo clinical evaluations to verify its effectiveness. We sincerely hope that the establishment of appropriate treatment guidelines will lead to improved outcomes for patients with ischemic stroke as a complication of ATAAD.

Acknowledgements This study was conducted with funding from Intramural Research Funds for the Cardiovascular Diseases of National Cerebral and Cardiovascular Center (27-2-3 and 28-4-1).

\section{Compliance with ethical standards}

Conflict of interest There is no conflict of interest to disclose pertaining to the publication of this paper.

Open Access This article is distributed under the terms of the Creative Commons Attribution 4.0 International License (http://creativeco mmons.org/licenses/by/4.0/), which permits unrestricted use, distribution, and reproduction in any medium, provided you give appropriate credit to the original author(s) and the source, provide a link to the Creative Commons license, and indicate if changes were made.

\section{References}

1. Shinohara Y, Minematsu K. Cautions of aortic dissection in intravenous thrombolytic therapy with alteplase. Jpn J Stroke (Japanese). 2008;30:443-4.
2. Minematsu K, Toyoda K, Hirano T, Kimura K, Kondo R, Mori E, et al. Guidelines for the intravenous application of recombinant tissue-type plasminogen activator (alteplase), the second edition, October 2012: a guideline from the Japan Stroke Society. J Stroke Cerebrovasc Dis. 2013;22(5):571-600.

3. Koga M, Iguchi Y, Ohara T, Tahara Y, Inoue Y, Fukuda K, et al. Acute ischemic stroke caused by Stanford type A acute aortic dissection. Jpn J Stroke (Japanese). 2017. https://doi.org/10.3995/ jstroke.10592.

4. JCS Joint Working Group. Guidelines for diagnosis and treatment of aortic aneurysm and aortic dissection (JCS 2011): digest version. Circ J. 2013;77(3):789-828.

5. Committee for Scientific Affairs, The Japanese Association for Thoracic Surgery, Masuda M, Okumura M, Doki Y, Endo S, Hirata Y, Kobayashi J, Kuwano H, Motomura N, Nishida H, Saiki Y, Saito A, Shimizu H, Tanaka F, Tanemoto K, Toh Y, Tsukihara H, Wakui S, Yokomise H. Thoracic and cardiovascular surgery in Japan during 2014: annual report by the Japanese Association for Thoracic Surgery. Gen Thorac Cardiovasc Surg. 2016;64(11):665-97.

6. Doroghazi RM, Slater EE, DeSanctis RW, Buckley MJ, Austen WG, Rosenthal S. Long-term survival of patients with treated aortic dissection. J Am Coll Cardiol. 1984;3(4):1026-34.

7. Mehta RH, Suzuki T, Hagan PG, Bossone E, Gilon D, Llovet A, et al. Predicting death in patients with acute type a aortic dissection. Circulation. 2002;105(2):200-6.

8. Kawahito K, Adachi H, Yamaguchi A, Ino T. Early and late surgical outcomes of acute type A aortic dissection in patients aged 75 years and older. Ann Thorac Surg. 2000;70(5):1455-9.

9. Miller DC, Mitchell RS, Oyer PE, Stinson EB, Jamieson SW, Shumway NE. Independent determinants of operative mortality for patients with aortic dissections. Circulation. 1984;70(3 Pt 2):I153-64.

10. Haverich A, Miller DC, Scott WC, Mitchell RS, Oyer PE, Stinson EB, et al. Acute and chronic aortic dissections-determinants of long-term outcome for operative survivors. Circulation. 1985;72(3 Pt 2):II22-34.

11. Pansini S, Gagliardotto PV, Pompei E, Parisi F, Bardi G, Castenetto E, et al. Early and late risk factors in surgical treatment of acute type A aortic dissection. Ann Thorac Surg. 1998;66(3):779-84.

12. Sabik JF, Lytle BW, Blackstone EH, McCarthy PM, Loop FD, Cosgrove DM. Long-term effectiveness of operations for ascending aortic dissections. J Thorac Cardiovasc Surg. 2000;119(5):946-62.

13. Nienaber CA, Eagle KA. Aortic dissection: new frontiers in diagnosis and management: part I: from etiology to diagnostic strategies. Circulation. 2003;108(5):628-35.

14. Kawahito K, Adachi H, Yamaguchi A, Ino T. Preoperative risk factors for hospital mortality in acute type A aortic dissection. Ann Thorac Surg. 2001;71(4):1239-43.

15. Lai DT, Robbins RC, Mitchell RS, Moore KA, Oyer PE, Shumway NE, et al. Does profound hypothermic circulatory arrest improve survival in patients with acute type a aortic dissection? Circulation. 2002;106(12 Suppl 1):I218-28.

16. Hagan PG, Nienaber CA, Isselbacher EM, Bruckman D, Karavite DJ, Russman PL, et al. The International Registry of Acute Aortic Dissection (IRAD): new insights into an old disease. JAMA. 2000;283(7):897-903.

17. Bossone E, Corteville DC, Harris KM, Suzuki T, Fattori R, Hutchison S, et al. Stroke and outcomes in patients with acute type A aortic dissection. Circulation. 2013;128(11 Suppl 1):S175-9.

18. Gaul C, Dietrich W, Friedrich I, Sirch J, Erbguth FJ. Neurological symptoms in type A aortic dissections. Stroke. 2007;38(2):292-7. 
19. Ohara T, Koga M, Tokuda N, Tanaka E, Yokoyama H, Minatoya $\mathrm{K}$, et al. Rapid identification of type A aortic dissection as a cause of acute ischemic stroke. J Stroke Cerebrovasc Dis. 2016;25(8):1901-6.

20. Sakamoto Y, Koga M, Ohara T, Ohyama S, Matsubara S, Minatoya $\mathrm{K}$, et al. Frequency and detection of stanford type A aortic dissection in hyperacute stroke management. Cerebrovasc Dis. 2016;42(1-2):110-6.

21. Hama Y, Koga M, Tokunaga K, Takizawa H, Miyashita K, Iba Y, et al. Carotid ultrasonography can identify stroke patients ineligible for intravenous thrombolysis therapy due to acute aortic dissection. J Neuroimaging. 2015;25(4):671-3.

22. Iguchi Y, Kimura K, Sakai K, Matsumoto N, Aoki J, Yamashita S, et al. Hyper-acute stroke patients associated with aortic dissection. Intern Med. 2010;49(6):543-7.

23. Ehrlich MP, Ergin MA, McCullough JN, Lansman SL, Galla JD, Bodian CA, et al. Results of immediate surgical treatment of all acute type A dissections. Circulation. 2000;102(19 Suppl 3):III $248-52$.

24. Trimarchi S, Nienaber CA, Rampoldi V, Myrmel T, Suzuki $\mathrm{T}$, Mehta RH, et al. Contemporary results of surgery in acute type A aortic dissection: the International Registry of Acute Aortic Dissection experience. J Thorac Cardiovasc Surg. 2005;129(1):112-22.
25. Chiappini B, Schepens M, Tan E, Dell'Amore A, Morshuis W, Dossche K, et al. Early and late outcomes of acute type A aortic dissection: analysis of risk factors in 487 consecutive patients. Eur Heart J. 2005;26(2):180-6.

26. Ueyama K, Otaki K, Koyama M, Kamiyama H. Urgent simultaneous revascularization of the carotid artery and ascending aortic replacement for type A acute aortic dissection with cerebral malperfusion. Gen Thorac Cardiovasc Surg. 2007;55(7):284-6.

27. Gaul C, Dietrich W, Erbguth FJ. Neurological symptoms in aortic dissection: a challenge for neurologists. Cerebrovasc Dis. 2008;26(1):1-8.

28. Yoshimuta T, Yokoyama H, Okajima T, Tanaka H, Toyoda K, Nagatsuka K, et al. Impact of elevated D-dimer on diagnosis of acute aortic dissection with isolated neurological symptoms in ischemic stroke. Circ J. 2015;79(8):1841-5.

29. Matsubara S, Koga M, Ohara T, Iguchi Y, Minatoya K, Tahara Y, et al. Cerebrovascular imaging of cerebral ischemia in acute type A aortic dissection. J Neurol Sci. 2018;388:23-7.

30. Tokuda N, Koga M, Ohara T, Minatoya K, Tahara Y, Higashi $\mathrm{M}$, et al. Urgent detection of acute type A aortic dissection in hyperacute ischemic stroke or transient ischemic attack. J Stroke Cerebrovasc Dis. 2018. https://doi.org/10.1016/j.jstrokecerebrov asdis.2018.03.010. 